\title{
Mental Size Scaling of Three-Dimensional Objects Perceived Visually or Tactilely
}

\author{
Magdalena Szubielska' and Bibianna Bałaj ${ }^{2}$ \\ 'The John Paul II Catholic University of Lublin, Institute of Psychology, Lublin, Poland \\ ${ }^{2}$ Nicolaus Copernicus University in Toruń, Faculty of Humanities, Deparment of Psychology, Toruń, Poland
}

\section{KEYWORDS}

visual perception, tactile perception, imagery debate, scaling time, size estimation
ABSTRACT

The issue of pictorial or propositional nature of imagery remains unresolved. To take a step forward into the debate, we conducted a systematic evaluation of time and accuracy of mental scaling in sighted people. Participants viewed or touched three-dimensional objects and then had to imagine them in a resized version, depending on a given scale. Both the mental scaling time and the estimated object size were measured. To promote verbal or perceptual strategies, the size was estimated verbally or bimanually, respectively. It was found that time taken for mental scaling is a linear function of decreasing and increasing scale and that the modality of perception did not influence the time taken to perform the operation. The results contribute to the knowledge of object size estimation by revealing the interaction between the modality of the object perception and the accuracy of size estimation by sighted adults. The accuracy of estimation was greater when the imagery representation was based on visual rather than tactile perception, but only in the case of verbal size assessment. Verbal height estimation in centimeters showed a tendency towards underestimation, while bimanually estimated sizes tended to be overestimated. The results indicate that people can use pictorial as well as prepositional strategies, depending on the task.

\section{INTRODUCTION}

Mental scaling involves a cognitive simulation of changing the size of a representation of spatial stimuli. Two explanations of this process were identified in the literature: (a) coding of relative distances (propositional) and (b) perception-based reasoning (pictorial). According to the first explanation, people encode spatial information by establishing relations between elements in a space (e.g., by remembering that point $\mathrm{C}$ is located halfway between points $\mathrm{A}$ and $\mathrm{B}$ ). Therefore, changing the scale does not require any mental operations associated with size transformation (see Huttenlocher, Newcombe, \& Vasilyeva, 1999). In the perception-based approach (proposed by Vasilyeva \& Huttenlocher, 2004), the process of mental scaling is explained through the metaphor of a magnifying glass or zooming in on a web map. Time taken to transform an image increases with the growing difference in size between the compared objects. According to the second approach, the result of the transformation is not perfect-for larger size changes, mental representations become imprecise (Möhring, Newcombe, \& Frick, 2014, 2016).

Both of these approaches can be considered in the classical context of the debate over the propositional versus analogue/pictorial nature of mental imagery. The topic has been continuously disputed since the 1970s between Kosslyn (see, e.g., 1973, 1983, 2005) and Pylyshyn (see, e.g., 1973, 1981, 2002; cf. e.g., Slezak, 2002). In an analogue imagery

Corresponding author: Magdalena Szubielska, The John Paul II Catholic University of Lublin, Institute of Psychology, Al. Racławickie 14, 20-950 Lublin. E-mail:magdasz@kul.pl 
strategy, a functional equivalence between perception and imagination was identified in the processes of mental rotation (Shepard \& Metzler, 1971) and mental scanning (Kosslyn, 1975). One of the objections against the concept of the pictorial nature of mental imagery is based on the fact that the results of studies allegedly confirming the concept stem from the design of the experimental procedure or the instructions that encouraged the subjects to apply the visualisation strategy.

\section{Time of Imagery Operation}

A number of studies using various types of two- and three-dimensional objects, viewed visually and tactilely, have observed recognition time to increase with increasing object size (Bennett \& Warren, 2002; Besner \& Coltheart, 1976; Bundesen \& Larsen, 1975; Craddock \& Lawson, 2009a, 2009b; Jolicoeur, 1987; Larsen \& Bundesen, 1978; Muthukumaraswamy, Johnson, \& Hamm, 2003; Szubielska, 2015). When drawings of a single figure were presented visually, recognition time was a roughly linear function of the proportion of the size of the test and reference figures (Larsen \& Bundesen 1978; see also Bundesen \& Larsen, 1975). Similar conclusions were reached in experiments with drawings of broken lines (Besner \& Coltheart, 1976), geometric figures (Muthukumaraswamy et al. 2003), and sketches of both natural objects and unknown shapes (Jolicoeur, 1987). In all of the above studies, the visual stimuli were presented on a blank background. Bennett and Warren (2002) also visually presented geometrical figures, but against a complex background that included monocular depth cues. The time taken to compare the shapes placed on this background increased with increasing difference from their so-called environmental sizecalculated from the angular size of an object and (as suggested by the pseudo three-dimensional background) its distance from the observer. Szubielska (2015), using embossed drawings, found that regardless of the complexity of the figures, blindfolded sighted participants needed more time to recognise an enlarged version compared to an unchanged figure. Craddock and Lawson (2009a, 2009b), in experiments on tactile and visual recognition of objects, found that in visual as well as in tactile cognition, changing the size of test objects slowed down their recognition time. Across these experiments, the reaction times to complete the mental visual task increased or decreased based on the size of the object, which supports an analogue approach. However, the results of these studies may be associated with the test procedure applied-in all the tests, stimuli of two sizes were looked at or touched (consecutively or simultaneously), and their sizes were then to be compared.

We formulated a question of whether or not, in an experiment where the participants cannot experience a stimulus that changes its size, we will also observe longer reaction times for larger mental size change scales of spatial stimuli. The positive answer would constitute an argument in favour of the perception-based explanation as being more probable than the judgment-based (propositional) explanation. The proposed procedure was inspired by an experiment where the participants (visually impaired individuals) were asked to imagine an object they had touched before and assess its size bimanually on a given scale using a string (see Szubielska \& Marek, 2015). In this procedure, when the participants placed their hands in the desired position, they received proprioceptive feedback. It seemed that in this way, participants could be encouraged to use analogue-based perception strategies. For this reason, we implemented a new assessment condition where the size estimation was given verbally, in centimeters. Moreover, the previous study examined a selective visually impaired group, whereas ours concerns the general population.

\section{Accuracy of Imagery Operations}

The accuracy of the perception-based approach in the mental scaling process is further supported by the results of experiments on the perception of stimuli presented on a changed scale. It has been reported that a change in visually perceived object size usually contributes to a decrease in recognition accuracy (Craddock \& Lawson, 2009a, 2009b; Jolicoeur, 1987; Möhring et al., 2014, 2016; Srinivas, Greene, \& Easton, 1997). This effect was not found in the study on the recognition of tactile figure drawings where two sizes of stimuli were compared. Perhaps the difference in size of stimuli was too small to reveal the difference in the accuracy of perception (Szubielska, 2015). In turn, Craddock and Lawson (2009a), in a study on the visual and tactile perception of objects, found a decrease in object recognition resulting from changes in their size under both perception conditions.

Based on a review of recent studies, Lacey and Sathian (2014) concluded that visual and haptic unisensory object processing show many similarities, and that similar unisensory visual and haptic representations lead to a shared multisensory representation (with a common neural substrate). The research on multisensory cognition suggests that, based on the visual and haptic perception, equally accurate imagery representations of objects can be created (e.g., Ernst, Lange, \& Newell, 2007; Norman, Norman, Clayton, Lianekhammy, \& Zielke, 2004; see also Craddock and Lawson, 2009a, for a contrary result), and taking into consideration the concept of a shared multisensory representation, we may predict that errors in the assessment of mentally enlarged and shrunk objects in different scales would not differ. To our best knowledge, the influence of costs of the scale change of a spatial stimulus size on the accuracy of its representation has not been researched very systematically (i.e., by comparisons of gradually decreasing and increasing stimuli), with one exception, and only for visual perception (Möhring et al., 2014). In order to systematically compare the costs of mental scaling of visually and tactilely perceived stimuli, we researched both perception conditions (visual and tactile) and five different size scale conditions.

A new element introduced in our research on the mental scaling by normally sighted individuals was the new way of inference about the error size, based on the estimation of an object imagined by the participants. In previous research, the accuracy of the size estimation of visually perceived lines, performed either verbally (in inches) or manually (by placing markers), did not differ (Schiffman, 1973). To our best knowledge, similar comparisons (i.e., of the accuracy of verbal vs. manual estimation) have not been conducted for haptically perceived objects in verbal versus pictorial strategies of solving the imagery task.

The expectations regarding the performance of size evaluation may be partially accounted for by reference to the ecological perspec- 
tive (Chang Wade, Stoffregen, \& Ho, 2008; see also Gibson \& Pick, 2000). In accordance with this perspective, the experience acquired at performing similar tasks in the past is a key factor in the accuracy of size assessment. Sighted individuals usually measure size using a measuring tape or a ruler. From the moment they learn to use a ruler at school, sighted people have frequent experience of measuring the size of objects or distances in centimeters or meters. This experience increases with age. Smith, Franz, Joy, and Whitehead (2005) examined the bimanual size estimation of known food products and found that their magnitude was overestimated by sighted adults in both visual and tactile exposures (estimations of the size of previously seen and touched objects did not differ), while blind adults assessed the size bimanually more accurately than did the sighted participants. When sighted adults estimated verbally (most likely in inches or centimeters) the size of previously seen objects, first with their eyes closed and then with eyes open, the accuracy of both estimation methods was very highly correlated (Bolles \& Bailey, 1956). This suggests that the verbal estimation of the magnitude of objects based on perceived visual cues and on memory could be equally accurate.

In our study, we took the perception-based approach and assumed the occurence of mental transformation (Möhring et al, 2014, 2016). Thus, we expected a linear increase in time taken to imagine an object and in errors in magnitude estimations with increasing or decreasing scaling factors. In accordance with the ecological perspective to perception and size estimation, we expected that bimanual estimation of the size of the imagined objects would not differ between visual and tactile perception, and that verbal estimation of the magnitude (in centimeters) would be more accurate when objects were perceived visually rather than tactilely. Additionally, we explored the types of errors (overestimation vs. underestimation) made in the case of manual and verbal estimation, as well as their dependency on the size of the object evaluated.

\section{METHOD}

\section{Participants}

A total of 42 students aged between 19 and 23 years $\left(M_{\text {age }}=19.80\right.$; $S D=0.87)$ participated in the study, 41 of whom were included in the analysis (40 women and one man; one woman was rejected because of noncompliant behaviour). Participants were randomly assigned to one of the four experimental groups: (a) visual perception, bimanual size estimation ( $n=10)$; (b) visual perception, verbal size estimation ( $n=10)$; (c) tactile perception, bimanual size estimation $(n=10)$; and (d) tactile perception, verbal size estimation $(n=11$, including one male).

\section{Materials}

All the materials used in the experiment were selected from Lego Duplo blocks. Four animal models (penguin, cat, giraffe, and hen), four blocks (black, brown, yellow, and white), and a square plate measuring $13 \mathrm{~cm}$ were used (see Figure 1). The height of the individual blocks was as follows: penguin $=6.2 \mathrm{~cm}$, cat $5.5=\mathrm{cm}$, giraffe $=10 \mathrm{~cm}$, hen $=4.4$ $\mathrm{cm}$, black block $=2.4 \mathrm{~cm}$, brown block $=4.4 \mathrm{~cm}$, yellow block $=4.4 \mathrm{~cm}$, and white block $=3.4 \mathrm{~cm}$. In addition, during the explanation stage of the study, a figure of a dog and an additional block were presented to participants. A thin, unstretchable string and a millimeter tape were used for the bimanual size estimations, and in all cases, a stopwatch was used to measure time.

\section{Procedure}

The task started with the visual or tactile perception of a single threedimensional object attached to the centre of the square board. The participants sat at a table with the board. Under the tactile condition, the participants had their eyes closed all the time, while in the visual condition, they opened and closed them according to the experimenter's signal. Visual exposure time was $10 \mathrm{~s}$, as measured from the moment of the participants opening their eyes (the open signal), after which the participants closed their eyes (the close signal). Touch exposure time was $20 \mathrm{~s}$ (twice as long as for visual perception, in accordance with the procedure developed by Ernst et al., 2007), measured from the time the participants started touching the object. After $20 \mathrm{~s}$, the experimenter said "stop," at which point the participants immediately let go of the object. Under both perception conditions, active exploration of the object was encouraged-under tactile conditions by touching it, and under visual conditions by manipulating the board to which the object was attached.

Next, the participants were asked to imagine the object at a given scale $(1: 3,1: 2,1: 1,2: 1$, or $3: 1)$. The instruction for the participants was „Now, imagine this object [...]" and "and imagine it being shrunk three times in size" (i.e., 1:3 scale), "shrunk twice in size" (1:2 scale), "at its original size" (1:1 scale), "enlarged twice in size" (2:1 scale), or "enlarged three times in size" (3:1 scale). The task began with a "start" signal, at which point the experimenter began measuring the par-

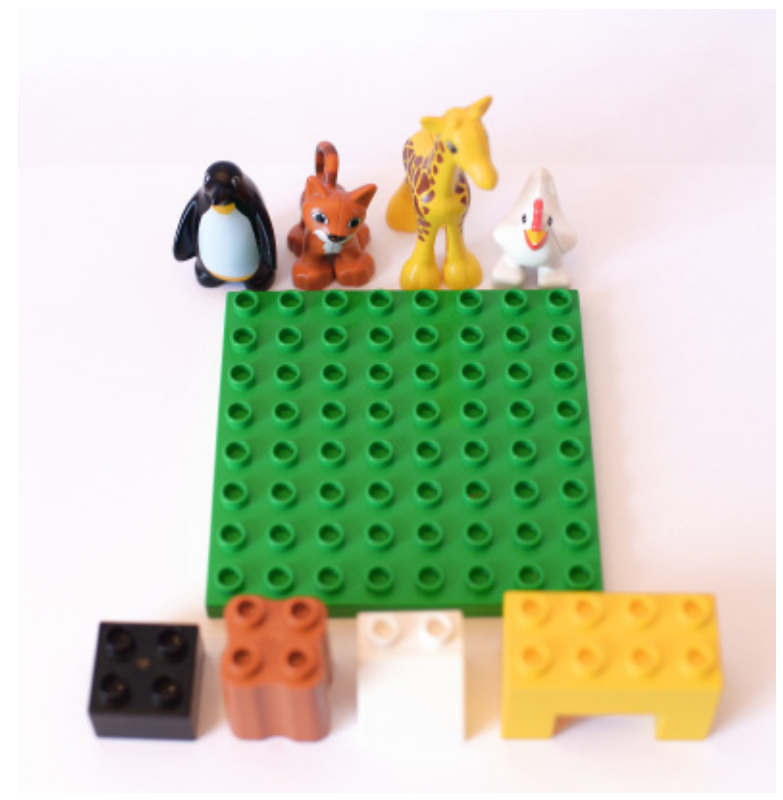

FIGURE 1.

Research materials. 
ticipants' task execution time. Imagining time was measured until the subject said "ready," which meant that he/she had imagined the object.

Participants were then asked to estimate the height of the objects they had imagined on a given scale. Answers for height estimation were given either verbally (in centimeters), or by indicating it bimanually using a string. The latter method followed the measurement procedure for estimating the size of objects used by Szubielska and Marek (2015), that is, the participants were asked to demonstrate the linear size of the imagined object on a piece of string. The relevant section of the string was then measured by the experimenter.

The experiment consisted of 40 trials (eight different shapes, five levels of scales), carried out in random order

\section{RESULTS}

\section{Time of Mental Scaling}

\section{TIME TO IMAGINE AN OBJECT IN RELATION TO SCALING FACTOR}

The analysis was performed after averaging the results obtained for the eight experimental objects. Because of the skewness in the reaction time distribution (see Table 1), reaction time values were subjected to a logarithmic transformation.

An analysis of variance (ANOVA) with repeated measures was performed for the time taken to imagine the objects (in seconds, transformed into logarithmic values). The within-subject factor was scale $(1: 3,1: 2,1: 1,2: 1,3: 1)$, the between-subject factors were modality (in which the stimulus was perceived, i.e, vision, touch) and the method of estimation (verbal, bimanual). A significant main effect of scale was found: $F(2.58,95.38)=79.23, p<.001, \eta_{\mathrm{p}}{ }^{2}=.68$. After carrying out Bonferroni corrected post hoc tests, it was found that the time taken to imagine objects on a 1:1 scale was significantly shorter than the time taken to imagine objects on a 1:2 $(p<.001), 1: 3(p<.001), 2: 1$ $(p<.001)$, and 3:1 $(p<.001)$ scale. In addition, a significantly shorter time for creating a mental image was found for 1:2 scale compared with 1:3 $(p<.001)$ scale, and for 2:1 scale compared with 3:1 scale $(p<.001$, see Figure 2). There were no significant differences in time taken to imagine objects between 1:3 and 3:1 scale $(p>.05)$ or 1:2 and $2: 1$ scale $(p>.05)$. The quadratic contrast was statistically significant, $F(1,37)=127.09, p<.001, \eta_{\mathrm{p}}^{2}=.78$.

The main effect of modality on time taken to imagine was not statistically significant, $F(1,37)=.48, p=.492$. The main effect method of estimation was also not statistically significant, $F(1,37)=.66, p=.422$.

The interaction between scale and method of estimation was significant, $F(2.58,95.38)=3.65, p=.02, \eta_{\mathrm{p}}^{2}=.09$. After conducting separate ANOVAs for verbal and bimanual methods of estimation and using Bonferroni corrected post hoc tests, it was found that in the verbal method of estimation condition, the time taken to imagine objects on a 2:1 scale was significantly shorter than the time taken to imagine objects on a 1:2 scale $(p=.012)$. No such effect was found in the bimanual method of estimation condition $(p>.05)$. The times to imagine were not significantly different for 1:3 and 3:1 scales, neither in the verbal nor the bimanual method of estimation ( $p>.05$ in each instance). In both the verbal and bimanual method of estimation conditions, the time taken to imagine objects on a 1:1 scale was significantly shorter than that taken to imagine objects on a 1:2 $(p<.001$ in both conditions), $1: 3$ ( $p<.001$ in both conditions), $2: 1$ ( $p<.001$ in both conditions), and 3:1 ( $p<.001$ in both conditions) scale; the time taken was also significantly shorter for 1:2 scale compared with 1:3 scale $(p<.001$ for the verbal and $p=.008$ in for the bimanual method of estimation condition), as well as for 2:1 scale compared with 3:1 scale ( $p=.005$ in the verbal and $p=.001$ in bimanual method of estimation condition, see Figure 3).

There were no more significant interactions (neither second nor third level) between the analysed factors ( $p>.05$ in all instances).

\section{Accuracy of Mental Scaling}

We tested the expectation that an error in magnitude estimation (a deviation from accurate size estimation) changes in relation to the scaling factor.

The estimation error (EE) was expressed as a percentage and calculated on the basis of the formula of $\mathrm{EE}=|(\mathrm{SEH}-\mathrm{CH}) / \mathrm{CH}| \times 100-$ where SEH stands for the height estimated by a subject (in centimeters) and $\mathrm{CH}$ is the correct height (in centimeters), that is, the object's actual height multiplied by the scale at which the object was to be imagined. The EE indicates the error rate percentage in relation to the correct size. The EE does not take into account whether an error of underestimation or overestimation was committed.

The analysis was performed after averaging the results obtained for the eight experimental objects (descriptive statistics are provided in Table 1). Because the EE measures had abnormal distribution and the variances in compared groups were not homogeneous, the conditions for a mixed ANOVA with a within-object factor of scale (1:3, 1:2, 1:1, $2: 1,3: 1$ ) and between-object factors of modality (visual, tactile) and method of estimation (verbal, bimanual) were not fulfilled. In this regard, the analysis-both parametric and nonparametric (depending on the condition of data distribution normality) were conducted separately in four experimental groups (visual perception, bimanual size estimation; visual perception, verbal size estimation; tactile perception, bimanual size estimation; and tactile perception, verbal size estimation).

For groups estimating the size verbally, a one-way ANOVA for the within-object factor of scale was performed. For the group that visually perceived stimuli and verbally estimated their size, no significant influence of scale was found, $F(2.26,20.32)=1.27, p=.307$. The influence of scale was also not statistically significant in the tactile perception and verbal size estimation group, $F(1.60,16.03)=2.33, p=.073$.

In the case of the groups estimating the size bimanually, nonparametric equivalents of ANOVA, that is, the Friedman tests, were performed for the within-object factor of scale. The statistically significant influence of scale was discovered both in the visual perception group, $\chi^{2}(4)=27.76, p<.001$, and in the tactile perception group, 
$\chi^{2}(4)=25.56, p<.001$. Pairwise comparisons were conducted using the Wilcoxon test.

For the visual perception and bimanual estimation group, a significantly larger EE (see Figure 4) was discovered for 1:3 than 1:2 scale $(z=-2.8, p=.005)$, for $1: 3$ than $1: 1(z=-2.8, p=.005), 1: 3$ than $2: 1$ $(z=-2.8, p=.005), 1: 3$ than $3: 1(z=-2.8, p=.005), 1: 2$ than $1: 1$ $(z=-2.29, p=.022), 1: 2$ than $2: 1(z=-2.7, p=.007), 1: 2$ than $3: 1$ $(z=-2.6, p=.009)$, and $1: 1$ than $2: 1(z=-2.19, p=.028)$. The estimation error made in 3:1 scale did not significantly differ from the error made in 2:1 scale $(z=-1.38, p=.169)$, or from the error made in 1:1 scale $(z=-1.78, p=.074)$.

For the tactile perception and bimanual estimation group, a significantly larger EE was found (see Figure 4) for 1:3 than 1:2 scale $(z=-2.7, p=.007)$, for $1: 3$ than $1: 1(z=-2.8, \mathrm{p}=.005), 1: 3$ than $2: 1$ $(z=-2.8, p=.005), 1: 3$ than $3: 1(z=-2.7, p=.007), 1: 2$ than $1: 1$ $(z=-2.7, p=.007), 1: 2$ than $2: 1(z=-2.6, p=.009), 1: 2$ than $3: 1$ $(z=-2.5, p=.013), 1: 1$ than $3: 1(z=-2.09, \mathrm{p}=.037)$, and $2: 1$ than $3: 1$ $(z=-2.19, p=.028)$. The estimation error made in $1: 1$ scale did not significantly differ from the error made in $2: 1$ scale $(z=-1.58, p=.114)$.

Next, we tested whether verbal and bimanual accuracy of the magnitude estimation is different for objects perceived visually or tactilely.

The conditions for the mixed ANOVA with a within-object factor of Scale (1:3, 1:2, 1:1, 2:1, 3:1) and between-object factors of modality (visual, tactile) and method of estimation (verbal, bimanual) have not been met. Analysing the data for verbal and bimanual methods of estimation separately, we found that the data obtained for EE satisfy the assumptions of the variance analysis only in the verbal method of estimation condition. For this condition, a mixed ANOVA with the within-subject factor of scale (1:3, 1:2, 1:1, 2:1, 3:1) and the between-subject factor of modality (vision, touch) was conducted. A significant main effect of modality was found, $F(1,19)=5.06$, $p=.037, \eta_{\mathrm{p}}{ }^{2}=.21$. In the verbal method of estimation condition, EE was greater in the condition where the object was previously touched rather than seen (see Figure 5). The main effect of scale, $F(2.16,41.11)=1.04$, $p=.368$, and the interaction of scale and modality, $F(2.16,41.11)=2.43$, $p=.097$, were not statistically significant. In the bimanual method of estimation condition, at each level of scale, we compared EE in groups of participants who perceived the objects either by sight or touch. Because EE measures had no normal distribution under the conditions of 2:1 and 3:1 scale, Mann-Whitney's $U$ tests were calculated. Modality did not significantly differentiate EE in the bimanual method of estimation condition in the case of $2: 1(z=-.38, p=.705)$ and 3:1 $(z=-.53$, $p=.597)$ scale. For the remaining levels of scale, $t$-tests were conducted. Modality did not significantly differentiate EE in the bimanual method of estimation also in the case of 1:3, $t(13.39)=.91, p=.377 ; 1: 2$, $t(13.48)=.49, p=.635$; or $1: 1, t(18)=.7, p=.493$ scale.

Futhtermore, we sought to examine what kind of errors are made with manual and verbal estimation and whether they depend on the size of the examined object.

The estimation error including the direction $\left(\mathrm{EE}_{\mathrm{D}}\right)$ was expressed as a percentage and calculated on the basis of the formula $\mathrm{EE}_{\mathrm{D}}=(\mathrm{SEH}$ - $\mathrm{CH}$ ) / CH $\times 100$-where SEH means the height estimated by the subject (in centimeters) and $\mathrm{CH}$ means the correct height (in centimeters), that is, the object's actual height, multiplied by the scale at which the object was to be imagined.

In the four experimental conditions and for each level of scale, $t$-tests for one trial were performed (when possible, i.e., when a condition of normal data distribution was met) by comparing EED to a 0 result, meaning an accurate size estimate (see Table 2). Significant errors of size underestimation were identified only in the case of verbal estimation, while for bimanual estimation, the significant errors were comprised of overestimation.

We also verified the $\mathrm{EE}_{\mathrm{D}}$ in size estimation of each stimulus in 1:1 scale, that is, without changing the size of the imagery (see Table 3). Since the requirements for mixed ANOVA for the within-object factor of object and between-objects of modality and method of estimation

\section{TABLE 1.}

Time Taken to Imagine Three-dimensional Objects at Various Scales (Expressed in Seconds, Averaged for Eight Experimental Objects): Mean ( $M$ ), SD, and Skewness (Sk.)

\begin{tabular}{|c|c|c|c|c|c|c|c|c|c|c|c|c|c|c|c|}
\hline \multirow{3}{*}{ Factors } & \multicolumn{6}{|c|}{ Verbal estimation } & \multicolumn{6}{|c|}{ Bimanual estimation } & \multirow{2}{*}{\multicolumn{3}{|c|}{$\begin{array}{l}\text { Without division into } \\
\text { groups }\end{array}$}} \\
\hline & \multicolumn{3}{|c|}{ Visual perception } & \multicolumn{3}{|c|}{ Tactile perception } & \multicolumn{3}{|c|}{ Visual perception } & \multicolumn{3}{|c|}{ Tactile perception } & & & \\
\hline & $M$ & $S D$ & Sk. & $M$ & $S D$ & $S k$. & $M$ & $S D$ & Sk. & $M$ & $S D$ & $S k$. & $M$ & $S D$ & $S k$. \\
\hline 1:3 Scale & 4.11 & 1.98 & .65 & 4.16 & 1.59 & .76 & .94 & 1.33 & .54 & 3.60 & 2.10 & 2.56 & 3.71 & 1.77 & 1.24 \\
\hline 1:2 Scale & 3.21 & 1.37 & .20 & 3.24 & 1.09 & .30 & 2.46 & 1.23 & 1.07 & 3.03 & 1.87 & 2.29 & 2.99 & 1.39 & 1.25 \\
\hline 1:1 Scale & 1.54 & .33 & -.07 & 1.92 & .67 & 1.24 & 1.80 & 1.02 & 1.76 & 2.00 & 1.17 & 2.18 & 1.82 & .84 & 2.12 \\
\hline 2:1 Scale & 2.96 & 1.57 & 1.78 & 2.90 & .98 & .21 & 2.77 & 1.49 & 1.44 & 3.07 & 2.34 & 2.80 & 2.92 & 1.60 & 2.33 \\
\hline 3:1 Scale & 3.81 & 1.79 & .48 & 3.61 & 1.58 & .37 & 3.68 & 2.07 & .91 & 3.91 & 2.74 & 2.55 & 3.75 & 2.00 & 1.59 \\
\hline
\end{tabular}




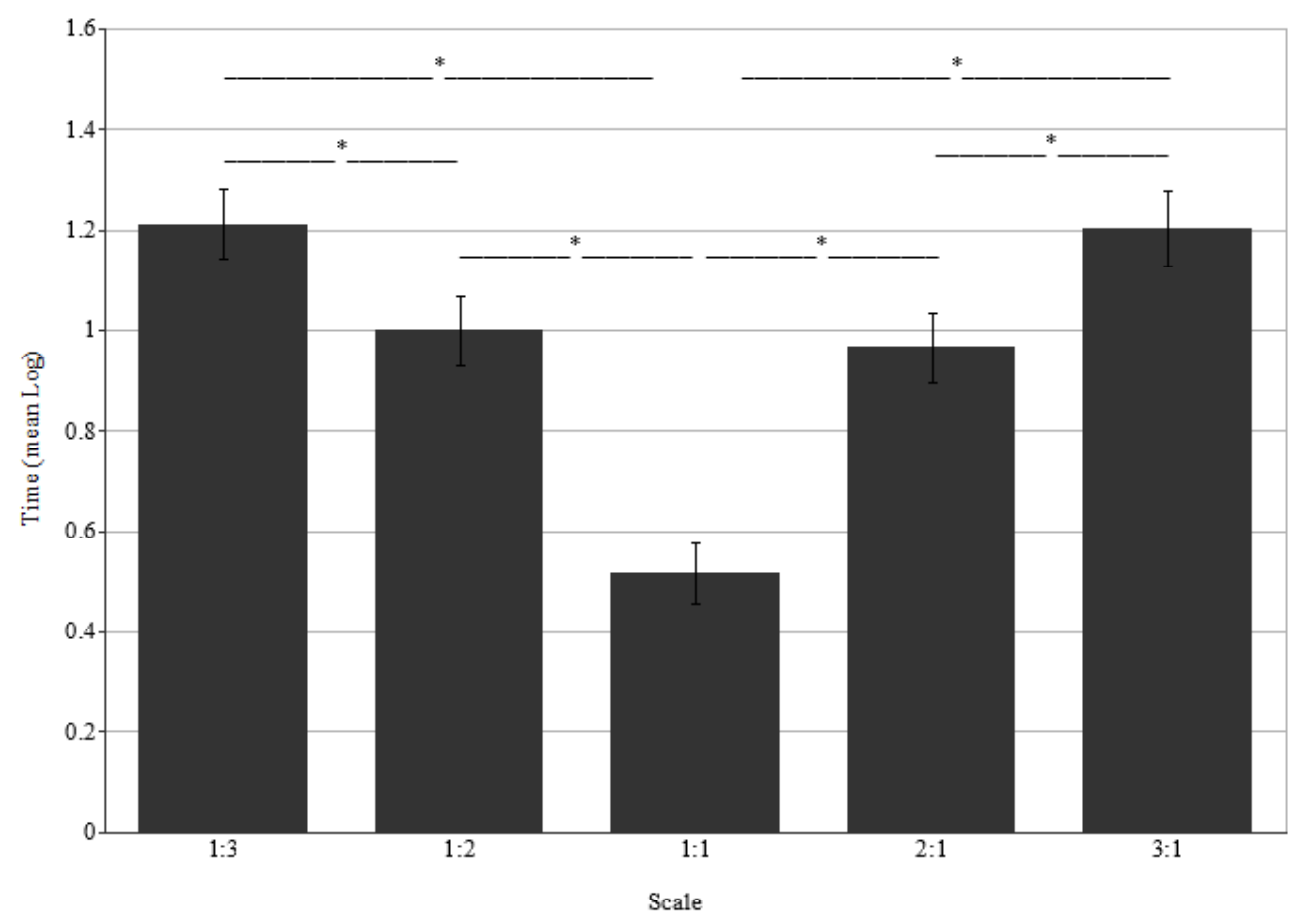

\section{FIGURE 2.}

Time (expressed in seconds, transformed into logarithmic values) taken to imagine the objects in various scales. Error bars represent the $95 \%$ Cl. Asterisks indicate significant differences.

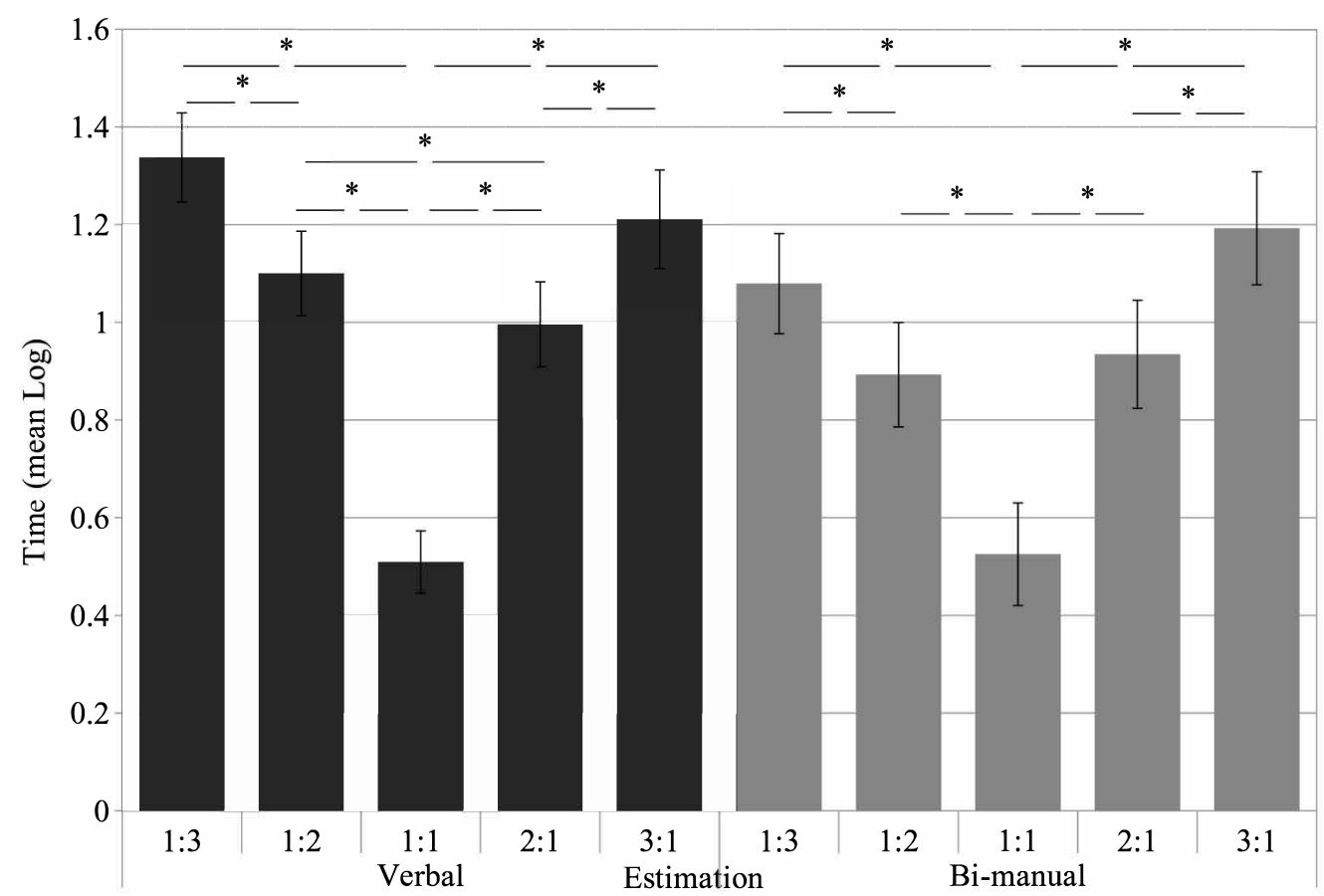

\section{FIGURE 3.}

Time (expressed in seconds, transformed into logarithmic values) taken to imagine the objects in various scales for verbal and bimanual estimation conditions. Error bars represent the $95 \%$ Cl. Asterisks indicate significant differences. 
were not met, we separately ran a one-way ANOVA with a repeated measure for the factor of object for each experimental group. The stimuli were introduced to the analysis in the growing order of size and semantics: (a) black block, (b) white block, (c) brown block, (d) yellow block, (e) hen, (f) cat, (g) penguin, and (h) giraffe.

In the condition of visual perception and verbal estimation of its size, a main effect of object was discovered, $F(7,63)=5.03, p<.001$, $\eta_{\mathrm{p}}{ }^{2}=.36$, and post hoc tests with the Bonferroni correction revealed that the size estimates for the hen brought significantly larger errors of underestimation than for the giraffe $(p=.016)$. Similarly, when a stimulus perceived tactilely and its size was then estimated verbally, the main effect of object, $F(2.34,23.38)=3.97, p=.028, \eta_{p}{ }^{2}=.28$, and a significantly greater error of underestimation was discovered for the hen than for the giraffe (see Table 3). In addition, the main effect of object was discovered for the condition of visual perception and bimanual estimation, $F(7,63)=4.13, p=.001, \eta_{\mathrm{p}}{ }^{2}=.31$, as well as for tactile perception and bimanual estimation, $F(7,63)=2.57, p=.021$, $\eta_{\mathrm{p}}{ }^{2}=.22$. For both these conditions, however, the post hoc tests with the Bonferroni correction did not reveal any statistically significant differences in estimation errors for the analyzed pairs of objects ( $p s>.05)$.

\section{DISCUSSION}

The main purpose of the current study was to systematically evaluate the time and accuracy of mental scaling of three-dimensional objects perceived visually or tactilely. The errors in the verbal (given in centimeters) or bimanual (performed with a string) estimation of the height of an object were used as the indicators of the accuracy of size scaling. The theoretical background of our study refers to the classical debate on the nature of imagery and the ecological approach to perception and size estimation. If the mental scaling functions similar to mental rotation, scaling time increasing as the scale increases is an argument for the pictorial nature of the imagery.

The first hypothesis, according to which the time taken to perform the mental scaling is a linear (either increasing or decreasing) scaling function, has been confirmed. The time required to create the mental image of a resized object was longer than in the control conditions that is, when the mental image of an object did not need to be resized. These results are consistent with other research on the visual and tactile recognition of objects presented in different scales (Bennett \& Warren, 2002; Craddock \& Lawson, 2009a, 2009b; Jolicoeur, 1987; Szubielska, 2015). Moreover, the time taken for mental scaling was a linear function of the changing size of an imagined object-this effect was found for mental processing of objects perceived both visually and tactilely. In previous studies, a similar effect was found only in the condition of visual perception of objects (Besner \& Coltheart, 1976; Bundesen \& Larsen, 1975; Larsen \& Bundesen, 1978; Muthukumaraswamy et al., 2003) and simple maps (Möhring et al., 2014, 2016). In our study, the time needed to complete the mental scaling operation was directly proportional to the change in scale, regardless of whether the imagined object was reduced or enlarged. This could constitute an argument in favour of the perception-based explanation (and the Kosslyn approach) over the analytical approach. However, the information about how the changed size is to be estimated influenced the time of the scaling operation (in interaction with the scaling factor). Most likely the estimation that is

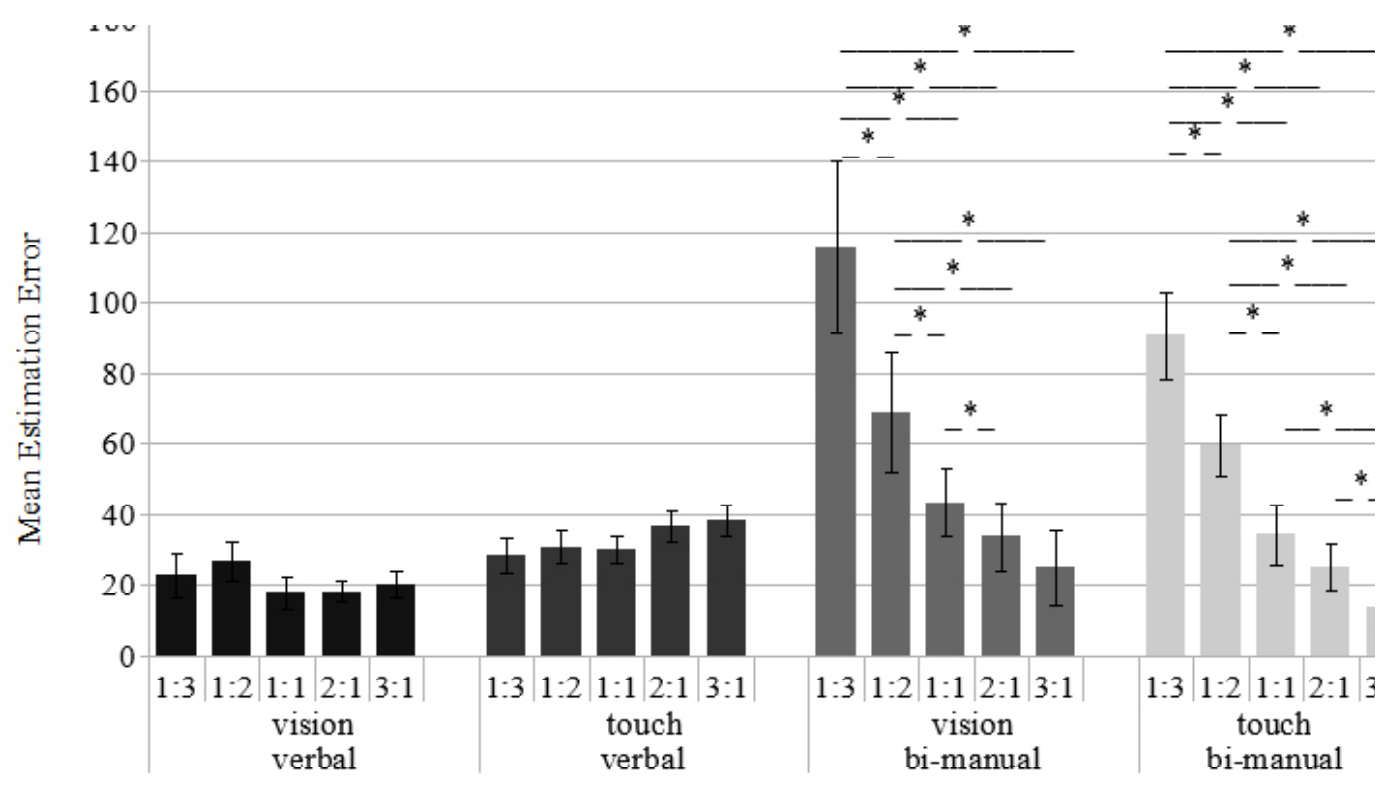

Estimation

FIGURE 4.

Absolute errors (EEs) in various scales for the vision and touch modality for the verbal and bimanual estimation groups. Error bars represent the $95 \% \mathrm{Cl}$. Asterisks indicate significant differences. 


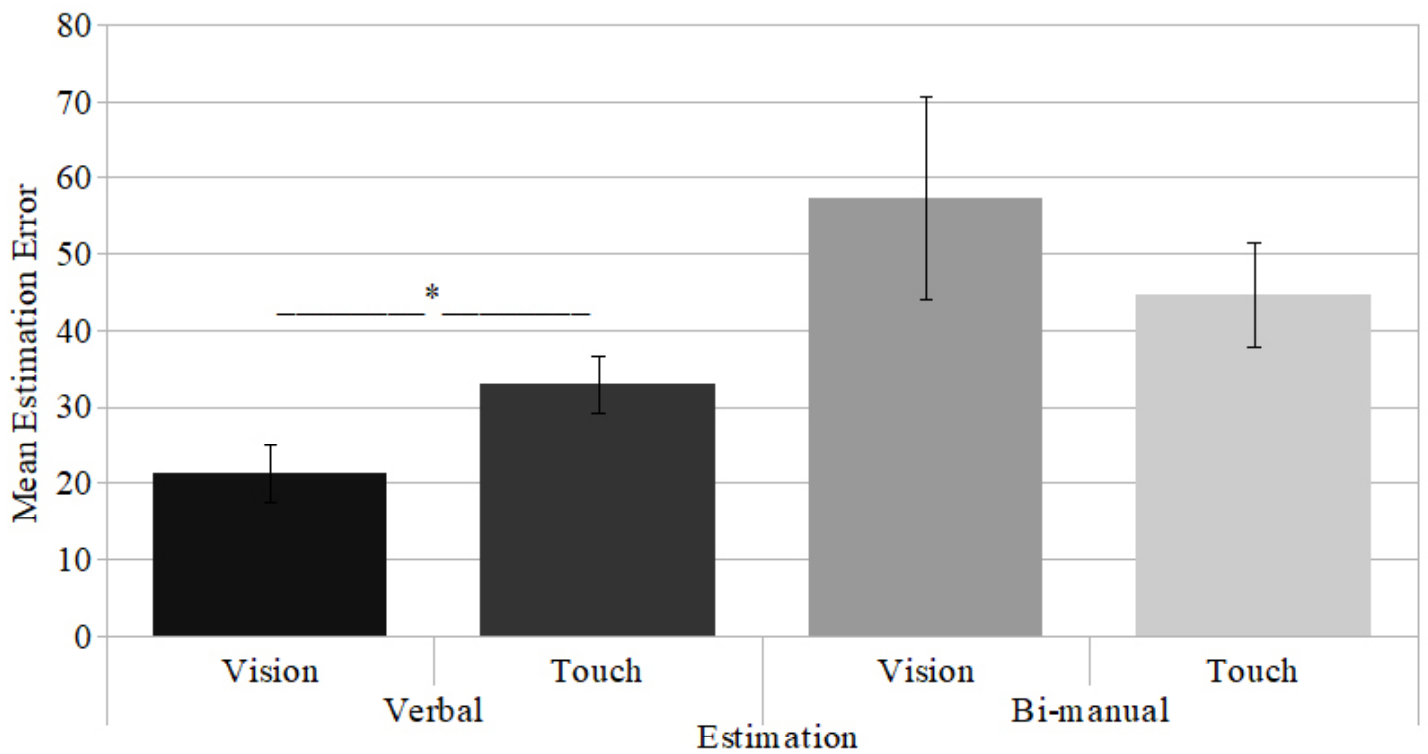

FIGURE 5.

Absolute errors (EEs) for the vision and touch modality for the verbal and bimanual estimation groups. Error bars represent the 95\% Cl. Asterisks indicate significant differences.

to be made verbally encourages the participants to apply the propositional strategy already at the stage of imagining the object. In the condition of a double size reduction, as compared with a double size increase, a significantly longer reaction time was observed only when the verbal estimation task was to be performed directly after the scaling operation - this difference was not observed when the following task comprised of a bimanual estimation. Besides, the descriptive statistics suggest the possible analogue direction of differences between the situation of triple reduction and enlargement of an object. It is possible that the differences were not revealed in our experiment due to the rather small number of participants. Multiplication is a mathematical operation generally performed faster than division (Rosenberg-Lee, Chang, Young, Wu, \& Menon, 2011), thus, it may be assumed with verbal size estimation being the following task, the participants were already getting ready for this task at the stage of scaling. It can be concluded that the estimation method encouraged the participants to apply a specific strategy of performing the imagery task: a mental transformation of an object when the following task involved a bimanual estimation or a mental transformation of an object assisted with a judgement-based strategy (associated with mathematical operations) when the following task was a verbal size estimation.

The second hypothesis, according to which the error in estimating the size of imagined objects is a linear function of reducing or enlarging the image, was not confirmed. This hypothesis was verified with an absolute (i.e., regardless of its direction) percentage of error as an error indicator. In the case of verbal estimation in centimeters, the error did not depend on the scale value at which the object was to be imagined, regardless of the modality of the object perception. Conversely, in the case of bimanual estimation-regardless whether an object was previously viewed or touched-the error size was inversely proportional to the scale value at which the object was to be imagined, which was particularly apparent in the case of decreasing sizes. The results suggest that the different estimation conditions encouraged the participants to apply different performance strategies for the task. When the size was to be estimated in centimeters, the arithmetical operation of multiplication or division was performed on the preliminarily estimated size of the object. Probably, the preliminary estimation took place at the stage of object perception, as confirmed by the estimation time for objects where the numerical value was to be multiplied or divided (c.f. Rosenberg-Lee et. al., 2011). These were fairly simple mathematical operations and their correct execution was easy for an adult, hence, there were no differences in the value of error made in the estimation of object sizes at different scales. This specific task, comprising of size estimation in centimeters, led the participants to use the propositional strategy and apply their previous knowledge, that is, to behave exactly as described by Pylyshyn when he wrote about the performance of imagery tasks $(1973,1981,2002)$. In turn, when the size was estimated bimanually, the participants could not effectively use the analytical strategy and most likely used only the analogue strategy (c.f. Kosslyn, $1973,1975,1983,2005)$-confirmed by the time taken to perform the scaling operation. However, it cannot definitely be concluded that the greater the scale change the greater the estimation error, as the supporters of the perception-based approach to the scaling explanation claim (c.f. Möhring et al., 2014, 2016; Vasilyeva \& Huttenlocher, 2004). Such an effect was observed only for the operation of a size decrease, which can be associated with the canonical size effect (Konkle \& Oliva, 2011) and with the difficulty of an accurate estimation of small objects by hand. Because the size of the mental representation is a logarithmic function of the size of the stimulus (cf. Konkle \& Oliva, 2011), a higher probability of making a greater error (understood as a percentage deviation from the correctly estimated size) exists for mental representations that are smaller. It was examined whether the greater difficulty in 
the bimanual size estimation of objects in smaller scales, rather than in bigger scales, results from small sizes of the objects or from the fact that the mental reduction of size is more difficult than mental enlarging. For this purpose, an additional analysis was performed comparing the error in size estimation of each stimulus in the condition where no scaling is required (1:1 scale). No differences in the error value were found for bimanual estimation of the experimental stimuli of different sizes. This leads to the conclusion that the difficulty with an accurate bimanual size estimation results from the difficulty of the mental size reduction operation rather than from the difficulty in size estimation of smaller and larger objects. It is, however, possible that the differences in difficulties with the size estimation of smaller and larger objects have not been revealed due to insufficient differences in stimuli size. This issue requires further research-a future study could include more differentiated scales and use a more precise measuring tool than a length of string or the participants' hands (e.g., a device similar to the one used by Chang et al., 2008), as well as stimuli of more diversified sizes.

The next expectation in this study was that the accuracy of the bimanual size estimation of the imagined objects would not differ regardless of whether the objects were previously perceived visually or tactilely and that the verbal size estimation (in centimeters) would be more accurate when objects were perceived visually rather than tactilely. This hypothesis has been fully confirmed. The error of estimation was greater in the verbal condition where an object had been previously touched rather than seen, and the perception modality did not significantly differentiate the error of estimation in the bimanual estimation of magnitude. This is probably due to the vast experience sighted people have with looking at centimeters on a ruler when taking measurements. The result obtained is consistent with other research results applying the ecological approach to the explanation of perception processes (cf. Gibson \& Pick, 2000), where the correctness of task performance was greater for participants who had previous experience with similar tasks from their everyday life (Chang et al., 2008).

Taking into consideration the direction of error revealed that while estimating the size verbally, we tend to underestimate and while estimating bimanually, to overestimate the size. Oculographic tests confirm that visual imagery representations are spontaneously reduced in size (Johansson, Holsanova, \& Holmqvist, 2006), which explains the underestimation in the verbal estimation conditions. Perhaps also for bimanual estimation, the imagery representation used by the participants was underestimated, but it was correlated with an overestimation error committed for a manual estimation that occurred in previous studies on size estimation (c.f. Ganel, Namdar, \& Mirsky, 2017).

In conclusion, following the mental scaling procedure of Szubielska and Marek (2015), our study confirms the results of other experiments on mental size scaling (Bennett \& Warren, 2002; Jolicoeur, 1987; Larsen \& Bundesen, 1978; Muthukumaraswamy et al., 2003; Srinivas et al., 1997; Szubielska, 2015). In these studies, as in ours, the imagining task took longer when it required mentally changing the size of the object than when the object did not need to be resized. The modality of perception-visual or tactile-did not affect the time taken to perform the mental scaling operation. The results concerning the value of the estimation error only partially confirm the perception-based explanation of mental scaling (Möhring et al., 2014, 2016; Vasilyeva \& Huttenlocher, 2004).

Our research has also shown that the type of task influences the participants' choice between the analogue and analytical strategy. In the context of the debate over the nature of imagery (Slezak, 2002), it allows us to conclude that people probably use both the analogue and propositional representations and, in a given situation, preference of one over the other depends on the type of task at hand. It was established that the accuracy of the imagery task performance may result from the everyday experience with performing similar tasks.

\section{TABLE 2.}

Averaged Estimation Error Including the Direction $\left(\mathrm{EE}_{\mathrm{D}}\right)$ : Mean $(M)$, SD, One Sample $\boldsymbol{t}$ Test

\begin{tabular}{|c|c|c|c|c|c|c|c|c|c|c|c|c|c|c|c|c|}
\hline \multirow{3}{*}{ Factors } & \multicolumn{8}{|c|}{ Verbal estimation } & \multicolumn{8}{|c|}{ Bimanual estimation } \\
\hline & \multicolumn{4}{|c|}{ Visual perception } & \multicolumn{4}{|c|}{ Tactile perception } & \multicolumn{4}{|c|}{ Visual perception } & \multicolumn{4}{|c|}{ Tactile perception } \\
\hline & $M$ & $S D$ & $t$ & $p$ & $M$ & $S D$ & $t$ & $p$ & $M$ & $S D$ & $t$ & $p$ & $M$ & $S D$ & $t$ & $p$ \\
\hline 1:3 Scale & -21.09 & 21.27 & -3.14 & $.012^{*}$ & -28.37 & 16.99 & -5.54 & $<.001^{* * *}$ & 115.78 & 77.56 & 4.72 & $.001^{\star *}$ & 9.63 & 39.55 & 7.25 & $<.001^{* * *}$ \\
\hline 1:2 Scale & -23.87 & 21.73 & -3.47 & $.007^{\star *}$ & -3.87 & 14.67 & -6.98 & $<.001^{\star * *}$ & 68.91 & 53.27 & 4.09 & $.003^{\star *}$ & 59.71 & 27.50 & 6.87 & $<.001^{\star * *}$ \\
\hline 1:1 Scale & -15.46 & 17.22 & -2.84 & $.019^{*}$ & -27.20 & 19.08 & - & - & 43.41 & 3.76 & 4.46 & $.002^{* *}$ & 34.32 & 27.23 & 3.99 & $.003^{* *}$ \\
\hline 2:1 Scale & -9.94 & 18.90 & -1.66 & .131 & -25.35 & 31.50 & - & - & 31.91 & 33.06 & 3.05 & $.014^{\star}$ & 2.57 & 25.40 & - & - \\
\hline 3:1 Scale & -13.19 & 19.84 & -2.10 & .065 & -29.07 & 29.91 & - & - & 17.59 & 38.71 & - & & 4.42 & 19.5 & .72 & .491 \\
\hline
\end{tabular}

Note. "-" means that the test was not calculated due to the lack of normality of the distribution.

${ }^{\star} p<.05 .{ }^{* *} p<.01 .{ }^{* *} p<.001$. 


\section{TABLE 3.}

Estimation Error Including the Direction $\left(\mathrm{EE}_{\mathrm{D}}\right)$ for Experimental Objects: Mean $(M)$ and $S D$

\begin{tabular}{|c|c|c|c|c|c|c|c|c|}
\hline \multirow{3}{*}{$\begin{array}{l}\text { Object (height of the } \\
\text { object in centimeters }\end{array}$} & \multicolumn{4}{|c|}{ Verbal estimation } & \multicolumn{4}{|c|}{ Bimanual estimation } \\
\hline & \multicolumn{2}{|c|}{ Visual perception } & \multicolumn{2}{|c|}{ Tactile perception } & \multicolumn{2}{|c|}{ Visual perception } & \multicolumn{2}{|c|}{ Tactile perception } \\
\hline & $M$ & $S D$ & $M$ & $S D$ & $M$ & $S D$ & $M$ & $S D$ \\
\hline Black block (2.4) & -22.08 & 17.68 & -33.90 & 24.08 & 62.08 & 40.42 & 50.42 & 42.69 \\
\hline White block (3.4) & -18.53 & 24.53 & -32.35 & 22.67 & 49.41 & 46.58 & 43.82 & 43.04 \\
\hline Brown block (4.4) & -1.59 & 23.35 & -27.69 & 29.70 & 61.36 & 43.36 & 33.86 & 45.84 \\
\hline Yellow block (4.4) & -6.82 & 34.78 & -5.58 & 42.29 & 65.00 & 47.07 & 48.64 & 37.62 \\
\hline Hen figurine (4.4) & -31.14 & 17.51 & -42.77 & 18.90 & 37.27 & 42.71 & 34.55 & 22.06 \\
\hline Cat figurine (5.5) & -29.10 & 14.08 & -36.86 & 24.92 & 27.82 & 41.51 & 19.45 & 25.90 \\
\hline Penguin figurine (6.2) & -6.45 & 26.12 & -23.46 & 26.47 & 19.52 & 26.74 & 17.42 & 31.36 \\
\hline Giraffe figurine (10) & -8.00 & 16.87 & -15.00 & 18.57 & 24.80 & 33.97 & 26.40 & 31.18 \\
\hline
\end{tabular}

\section{Limitations and Directions for Future Research}

Several limitations of our study could be identified. Firstly, the study did not control the way in which the participants tactilely perceived the stimuli. For instance, manipulating the stand may encourage mental rotation, whereas walking around the object may facilitate the creation of a different mental representation, for example, including the perspective. In future studies, the strategies of tactile perception should be categorised and verified as to how they translate into the time and accuracy of the scaling operation. Secondly, all of the animal shapes were larger than the geometric blocks. A difference was revealed in the error value of estimation of the hen and the giraffe (in both estimation conditions, for a 1:1 scale, perceived tactilely) — the hen, being the smallest figure, was underestimated more than the giraffe, being the largest figure. We are not able to determine if this effect is in any way associated with the semantics of the stimuli or rather with the difference in their size. In future studies, the semantics and sizes of the stimuli should be independently manipulated. In the future, the sizes of the stimuli and of the scales should be more diversified. Also, having adopted an ecological approach to perception and size evaluation as a background for the studies, our future experiments on tactile size perception and verbal or manual estimation should take into consideration the visual status of participants and include both sighted and blind individuals. Furthermore, a more precise size measurement tool should be used instead of hands and a string. Finally, in future research, some questions about the participants' strategy used during the task could be asked after the study.

\section{FOOTNOTES}

${ }^{1}$ The patterns in the data are unchanged when the analysis is conducted on reaction time before and after the logarithmic transformation.

\section{ACKNOWLEDGEMENTS}

The authors declare equal authorship of the article.

\section{REFERENCES}

Bennett, D. J., \& Warren, W. (2002). Size scaling: Retinal or environmental frame of reference? Perception \& Psychophysics, 64, 462-477. doi: 10.3758/BF03194718 www

Besner, D., \& Coltheart, M. (1976). Mental size scaling examined. Memory \& Cognition, 4, 525-531. doi: 10.3758/BF03213214 WWW

Bolles, R. C., \& Bailey, D. E. (1956). Importance of object recognition in size constancy. Journal of Experimental Psychology, 51, 222-225. doi: 10.1037/h0048080 |www

Bundesen, C., \& Larsen, A. (1975). Visual transformation of size. Journal of Experimental Psychology: Human Perception and Performance, 1, 214-220. doi: 10.1037/0096-1523.1.3.214 (www

Chang, C.-H., Wade, M. G., Stoffregen, T. A., \& Ho, H.-Y. (2008). Length perception by dynamic touch: The effects of aging and experience. The Journals of Gerontology: Series BPsychological Sciences and Social Sciences, 63, 165-170. doi: 10.1093/geronb/63.3.P165 WWW

Craddock, M., \& Lawson, R. (2009a). Size-sensitive perceptual representations underlie visual and haptic object recognition. 
PLoS One, 4, e8009. doi: 10.1371/journal.pone.0008009 $\overline{\mathrm{WWW}}$

Craddock, M., \& Lawson, R. (2009b). The effects of size changes on haptic object recognition. Attention, Perception, \&

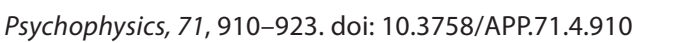

Ernst, M. O., Lange, C., \& Newell, F. N. (2007). Multisensory recognition of actively explored objects. Canadian Journal of Experimental Psychology, 61, 242-253. doi: 10.1037/ cjep2007025 $\underline{\underline{\omega W}}$

Ganel, T., Namdar, G., \& Mirsky, A. (2017). Bimanual grasping does not adhere to Weber's law. Scientific Reports, 7: 6467. doi: 10.1038/s41598-017-06799-4 WWW

Gibson, E. J., \& Pick, A. D. (2000). An ecological approach to perceptual learning and development. Oxford, England: Oxford University Press.

Huttenlocher, J., Newcombe, N. S., \& Vasilyeva, M. (1999). Spatial scaling in young children. Psychological Science, 10, 393-398. doi: 10.1111/1467-9280.00175

Johansson, R., Holsanova, J., \& Holmqvist, K. (2006). Pictures and spoken descriptions elicit similar eye movements during mental imagery, both in light and in complete darkness. Cognitive Science, 30, 1053-1079. doi: 10.1207/s15516709cog0000_86 WWW

Jolicoeur, P. (1987). A size-congruency effect in memory for visual shape. Memory \& Cognition, 15, 531-543. doi: 10.3758/ BF03198388 WWW

Konkle, T., \& Oliva, A. (2011). Canonical visual size for real-world objects. Journal of Experimental Psychology: Human Perception and Performance, 37, 23-37. doi:0.1037/a0020413 WWW

Kosslyn, S. M. (1973). Scanning visual images: some structural implications. Perception and Psychophysics, 14, 90-94. doi: 10.3758/BF03198621

Kosslyn, S. M. (1975). Information representation in visual images. Cognitive Psychology, 7, 341-370. doi: 10.1016/00100285(75)90015-8

Kosslyn, S. M. (1983). Ghosts in the mind's machine. Creating and using images in the brain. New York: Norton.

Kosslyn, S. M. (2005). Mental images and the brain. Cognitive Neuropsychology, 22, 333-347. doi: 10.1080/02643290442000130 [WWW

Lacey, S., \& Sathian, K. (2014). Visuo-haptic multisensory object recognition, categorization, and representation. Frontiers in Psychology, 5:730. doi: 10.3389/fpsyg.2014.00730 |wWw

Larsen, A., \& Bundesen, C. (1978). Size scaling in human pattern recognition. Journal of Experimental Psychology: Human

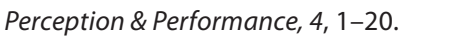

Muthukumaraswamy, S. D., Johnson, B. W., \& Hamm, J. P. (2003). A high density ERP comparison of mental rotation and mental size transformation. Brain and Cognition, 52, 271-280. doi: 10.1016/S0278-2626(03)00077-0 WWW

Möhring, W., Newcombe, N. S., \& Frick, A. (2014). Zooming in on spatial scaling: Preschool children and adults use mental transformations to scale spaces. Developmental Psychology, 50,
1614-1619. doi: 10.1037/a0035905 WWW

Möhring, W., Newcombe, N. S., \& Frick, A. (2016). Using mental transformation strategies for spatial scaling: Evidence from a discrimination task. Journal of Experimental Psychology: Learning, Memory, and Cognition, 42, 1473-1479. doi: 10.1037/

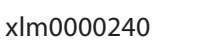

Norman, J. F., Norman, H. F., Clayton, A. M., Lianekhammy, J., \& Zielke, G. (2004). The visual and haptic perception of natural object shape. Perception \& Psychophysics, 66, 342-351. doi: 10.3758/BF03194883 WWW

Pylyshyn, Z. W. (1973). What the mind's eye tells the mind's brain: A critique of mental imagery. Psychological Bulletin, 80, 1-24. doi: $10.1037 / \mathrm{h} 0034650$

Pylyshyn, Z. W. (1981). The imagery debate: analogue versus tacit knowledge. Psychological Review, 88, 16-45. doi: 10.1037/0033295X.88.1.16

Pylyshyn, Z. W. (2002). Mental imagery: In search of a theory. Behavioral and Brain Sciences, 25, 157-238. doi: 10.1017/ S0140525X02000043 WWW

Rosenberg-Lee, M., Chang, T. T., Young, C. B., Wu, S., \& Menon, V. (2011). Functional dissociations between four basic arithmetic operations in the human posterior parietal cortex: A cytoarchitectonic mapping study. Neuropsychologia, 49, 2592-2608. doi: 10.1016/j.neuropsychologia.2011.04.035 WWW

Schiffman, H. R. (1973). A comparison of response modes on the perception of linear extent. Bulletin of the Psychonomic Society, 2, 276-278. doi: 10.3758/BF03329270

Shepard, R. N., \& Metzler, J. (1971, February). Mental rotation of three-dimensional objects. Science, 171(3972), 701-703. doi: 10.1126/science.171.3972.701 WWW

Slezak, P. (2002). The imagery debate: Déjà-vu all over again? Behavioral and Brain Sciences, 25, 209-210. doi: 10.1017/ S0140525X02490048

Smith, M., Franz, E. A., Joy, S. M., \& Whitehead, K. (2005). Superior performance of blind compared with sighted individuals on bimanual estimations of object size. Psychological Science, 16, 11-14. doi: 10.1111/j.0956-7976.2005.00773.x WwW

Srinivas, K., Greene, A. J., \& Easton, R. D. (1997). Visual and tactile memory for 2-D patterns: Effects of changes in size and leftright orientation. Psychonomic Bulletin \& Review, 4, 535-540. doi: 10.3758/BF03214345

Szubielska, M. (2015). Majoryzacja wyobrażeniowa figur poznawanych dotykowo przez osoby widzące i niewidome od urodzenia [Mental majorization of figures tactilely explored by sighted and congenitally blind individuals]. Roczniki Psychologiczne [Annals of Psychology], 18, 121-132. doi: 10.18290/rpsych.2015.18.1-5en

Szubielska, M., \& Marek, B. (2015). The role of visual experience in changing the size of objects in imagery processing. Journal of Visual Impairment \& Blindness, 109, 43-53.

Vasilyeva, M., \& Huttenlocher, J. (2004). Early development of scaling ability. Developmental Psychology, 40, 682-690. wWw 